\title{
Determination of synchronous generator nonlinear model parameters based on power rejection tests using a gradient optimization algorithm
}

\author{
A. BOBOŃ, A. NOCOŃ, S. PASZEK, and P. PRUSKI* \\ Institute of Electrical Engineering and Computer Science, Silesian University of Technology, 10 Akademicka St., 44-100 Gliwice, Poland
}

\begin{abstract}
The paper presents a method for determining electromagnetic parameters of different synchronous generator models based on dynamic waveforms measured at power rejection. Such a test can be performed safely under normal operating conditions of a generator working in a power plant. A generator model was investigated, expressed by reactances and time constants of steady, transient, and subtransient state in the $\mathrm{d}$ and $\mathrm{q}$ axes, as well as the circuit models (type $(3,3)$ and $(2,2)$ ) expressed by resistances and inductances of stator, excitation, and equivalent rotor damping circuits windings. All these models approximately take into account the influence of magnetic core saturation. The least squares method was used for parameter estimation. There was minimized the objective function defined as the mean square error between the measured waveforms and the waveforms calculated based on the mathematical models. A method of determining the initial values of those state variables which also depend on the searched parameters is presented. To minimize the objective function, a gradient optimization algorithm finding local minima for a selected starting point was used. To get closer to the global minimum, calculations were repeated many times, taking into account the inequality constraints for the searched parameters. The paper presents the parameter estimation results and a comparison of the waveforms measured and calculated based on the final parameters for $200 \mathrm{MW}$ and $50 \mathrm{MW}$ turbogenerators.
\end{abstract}

Key words: gradient optimization algorithm, synchronous generator models, parameter estimation, load rejection tests.

\section{Introduction}

The mathematical models and values of parameters of generating unit elements, primarily of synchronous generators, decide on the reliability of simulation investigations of power systems (PSs). Both simple and complex mathematical models of synchronous generators are used in these investigations. The models can be divided into XT-type models, expressed by standard parameters (reactances and time constants), and RL-type, expressed by resistances and inductances of electrical circuits. If the models are to accurately represent the phenomena occurring in a generator, saturation of the magnetic circuits [1] should be taken into account. It should be emphasized that for the analysis of transient states in a PS, nonlinear models are often used - without taking into account the saturation of the magnetic circuit [1-4]. The waveforms obtained this way (e.g. waveform of the generator terminal voltage) differ significantly from real ones, so the formulated conclusions (e.g. concerning the required protection automation or control systems) may be false.

The methods for determining electromagnetic parameters of synchronous generators are widely described and discussed in the scientific technical literature [2,5-11]. Among the measuring methods there are distinguished those based on the results of measurements at machine standstill (e.g. frequency methods

*e-mail: piotr.pruski@polsl.pl

Manuscript submitted 2016-11-15, revised 2017-01-20, initially accepted for publication 2017-02-16, published in August 2017.
SSFR $[2,6,7])$ and those based on the results of measurements at a rotating machine. The methods based on the measurement tests carried out under the conditions close to normal operation of a synchronous generator, such as power rejection tests $[2,7$, 11]. The load rejection test is of great practical importance. Its realization requires only minor changes in the normal operating procedures of a generating unit. The test can be carried out during a scheduled shutdown of the generator. More detailed description of the load rejection test is given in Section 3.

The paper presents the models of synchronous generators with a cylindrical rotor (turbogenerator) of type XT and RL that take into account the saturation of the machine's magnetic circuit. The parameter estimation method for these models is described. In this method, the dynamic waveforms of selected quantities measured during power rejection tests are used. The estimation results for $200 \mathrm{MW}$ and $50 \mathrm{MW}$ generators are given in the paper.

To determine the values of the parameters of the analysed mathematical models, the least squares method was used. An objective function was defined using the difference between the measured waveforms and simulation waveforms calculated with the use of the mathematical model. A widely used Newton gradient algorithm was used to minimize the objective function [12-15]. Section 5 contains a brief description of this algorithm together with its flow chart. This paper focuses on a broader description of the equations of individual models (Section 2). Moreover, the procedure for determination of initial conditions of simulations is thoroughly discussed (Section 4). It is a difficult issue, and mistakes made in this procedure result in errors in parameter estimation. 


\section{Synchronous generator models taking into account the main flux saturation effect}

2.1. The model of the type XT. The GENROU model with two equivalent circuits in the $\mathrm{d}$ and q generator axes is a typical representative of XT-type models of generators used widely for analyses of PSs and described in the literature [3, 10, 16-19]. In this model, transformation voltages in the generator armature circuits are neglected $[3,16,19-21]$.

The differential and algebraic equations in the $d$ axes of the GENROU model, expressed in relative units, have the form of $[3,18]$ :

$$
\begin{gathered}
V_{\mathrm{d}}=-\Psi_{\mathrm{q}}-I_{\mathrm{d}} R_{\mathrm{a}}=-\Psi_{\mathrm{q}}^{\prime \prime}+X_{\mathrm{q}}^{\prime \prime} I_{\mathrm{q}}-I_{\mathrm{d}} R_{\mathrm{a}}, \\
\frac{\mathrm{d} E_{\mathrm{q}}^{\prime}}{\mathrm{d} t}=\frac{1}{T_{\mathrm{do}}^{\prime}}\left(E_{\mathrm{fd}}-X_{\mathrm{ad}} I_{\mathrm{fd}}\right), \\
\frac{\mathrm{d} \Psi_{\mathrm{D} 1}}{\mathrm{~d} t}=\frac{1}{T_{\mathrm{do}}^{\prime \prime}}\left(E_{\mathrm{q}}^{\prime}-\Psi_{\mathrm{D} 1}-I_{\mathrm{d}}\left(X_{\mathrm{d}}^{\prime}-X_{\sigma}\right)\right), \\
\Psi_{\mathrm{d}}^{\prime \prime}=E_{\mathrm{q}}^{\prime} \frac{X_{\mathrm{d}}^{\prime \prime}-X_{\sigma}}{X_{\mathrm{d}}^{\prime}-X_{\sigma}}+\Psi_{\mathrm{D} 1} \frac{X_{\mathrm{d}}^{\prime}-X_{\mathrm{d}}^{\prime \prime}}{X_{\mathrm{d}}^{\prime}-X_{\sigma}} \\
X_{\mathrm{ad}} I_{\mathrm{fd}}=E_{\mathrm{q}}^{\prime}+\Delta\left(X_{\mathrm{ad}} I_{\mathrm{fd}}\right) \frac{\Psi_{\mathrm{d}}^{\prime \prime}}{\mid \Psi^{\prime \prime}}+{ }^{\prime}{ }^{\prime \prime} \\
+\left(X_{\mathrm{d}}-X_{\mathrm{d}}^{\prime}\right)\left(I_{\mathrm{d}}+T_{\mathrm{do}}^{\prime \prime} \frac{\mathrm{d} \Psi_{\mathrm{D} 1}}{\mathrm{~d} t} \frac{X_{\mathrm{d}}^{\prime}-X_{\mathrm{d}}^{\prime \prime}}{\left(X_{\mathrm{d}}^{\prime}-X_{\sigma}\right)^{2}}\right) .
\end{gathered}
$$

The differential and algebraic equations in the $\mathrm{q}$ axis are given by the formulas $[3,18]$ :

$$
\begin{gathered}
V_{\mathrm{q}}=\Psi_{\mathrm{d}}-I_{\mathrm{q}} R_{\mathrm{a}}=\Psi_{\mathrm{d}}^{\prime \prime}-X_{\mathrm{d}}^{\prime \prime} I_{\mathrm{d}}-I_{\mathrm{q}} R_{\mathrm{a}}, \\
\frac{\mathrm{d} E_{\mathrm{d}}^{\prime}}{\mathrm{d} t}=\frac{1}{T_{\mathrm{qo}}^{\prime}}\left(-I_{\mathrm{Q} 1} X_{\mathrm{aq}}\right), \\
\frac{\mathrm{d} \Psi_{\mathrm{Q} 1}}{\mathrm{~d} t}=-\frac{1}{T_{\mathrm{qo}}^{\prime \prime}}\left(-E_{\mathrm{d}}^{\prime}+\Psi_{\mathrm{Q} 1}+I_{\mathrm{q}}\left(X_{\mathrm{q}}^{\prime}-X_{\sigma}\right)\right), \\
\Psi_{\mathrm{q}}^{\prime \prime}=E_{\mathrm{d}}^{\prime} \frac{X_{\mathrm{q}}^{\prime \prime}-X_{\sigma}}{X_{\mathrm{q}}^{\prime}-X_{\sigma}}+\Psi_{\mathrm{Q} 1} \frac{X_{\mathrm{q}}^{\prime}-X_{\mathrm{q}}^{\prime \prime}}{X_{\mathrm{q}}^{\prime}-X_{\sigma}} \\
X_{\mathrm{aq}} I_{\mathrm{Q} 1}=E_{\mathrm{d}}^{\prime}+\Delta\left(X_{\mathrm{ad}} I_{\mathrm{fd}}\right) \frac{\Psi_{\mathrm{q}}^{\prime \prime}}{\mid \Psi_{\mathrm{aq}}^{\prime \prime}} \frac{X_{\mathrm{ad}}}{X_{\mathrm{q}}}{ }^{\prime \prime} \\
+\left(X_{\mathrm{q}}-X_{\mathrm{q}}^{\prime}\right)\left(I_{\mathrm{q}}+T_{\mathrm{qo}}^{\prime \prime} \frac{\mathrm{d} \Psi_{\mathrm{Q} 1}}{\mathrm{~d} t} \frac{X_{\mathrm{q}}^{\prime}-X_{\mathrm{q}}^{\prime \prime}}{\left(X_{\mathrm{q}}^{\prime}-X_{\sigma}\right)^{2}}\right) .
\end{gathered}
$$

The symbols used to denote the quantities and parameters in (1) and (2) are as follows: $V_{\mathrm{d}}, V_{\mathrm{q}}, I_{\mathrm{d}}, I_{\mathrm{q}}, \Psi_{\mathrm{d}}, \Psi_{\mathrm{q}}$ - stator winding voltages, currents and magnetic fluxes in the $\mathrm{d}$ and $\mathrm{q}$ axes, respectively; $\underline{\Psi}^{\prime \prime}=\Psi_{\mathrm{q}}^{\prime \prime}-\mathrm{j} \Psi_{\mathrm{d}}^{\prime \prime}-$ phasor of the subtransient flux linkage and its axial components; $\Psi_{\mathrm{D} 1}, \Psi_{\mathrm{Q} 1}, I_{\mathrm{Q} 1}-$ flux linkage and current in the first equivalent damping circuit in the $\mathrm{d}$ and $\mathrm{q}$ axes; $E_{\mathrm{d}}^{\prime}, E_{\mathrm{q}}^{\prime}-$ generator voltage in the $\mathrm{d}$ and $\mathrm{q}$ axes behind the transient reactance; $E_{\mathrm{fd}}, I_{\mathrm{fd}}$, - excitation voltage and exciting current; $R_{\mathrm{a}}, X_{\sigma}$ - leakage resistance and reactance of the stator; $X_{\mathrm{d}}, X_{\mathrm{q}}, X_{\mathrm{ad}}, X_{\mathrm{aq}}, X_{\mathrm{d}}^{\prime}, X_{\mathrm{q}}^{\prime}, X_{\mathrm{d}}^{\prime \prime}, X_{\mathrm{q}}^{\prime \prime}-$ synchronous, stator reaction, transient and subtransient reactance in the $\mathrm{d}$ and q axes, respectively; $T_{\mathrm{d} 0}^{\prime}, T_{\mathrm{d} 0}^{\prime \prime}, T_{\mathrm{q} 0}^{\prime}, T_{\mathrm{q} 0}^{\prime \prime}$ - transient and subtransient time constant in the $\mathrm{d}$ and $\mathrm{q}$ axes at the open winding of the generator stator. The term $\Delta\left(X_{\mathrm{ad}} I_{\mathrm{fd}}\right)$ expresses the effect of saturation of the magnetic cores.

The phenomenon of the machine magnetic circuit saturation [1] is taken into account in approximation, making it dependent on the magnetic flux linkages occurring behind the subtransient reactances in both axes $[2,3,17,18,20]$.

Figure 1 presents the nonlinear no-load characteristic of the generator (p.u.) expressed as a relationship between the subtransient flux linkage modulus and the excitation current. Based on this figure, the saturation coefficients $S_{10}$ and $S_{12}$ are

a)

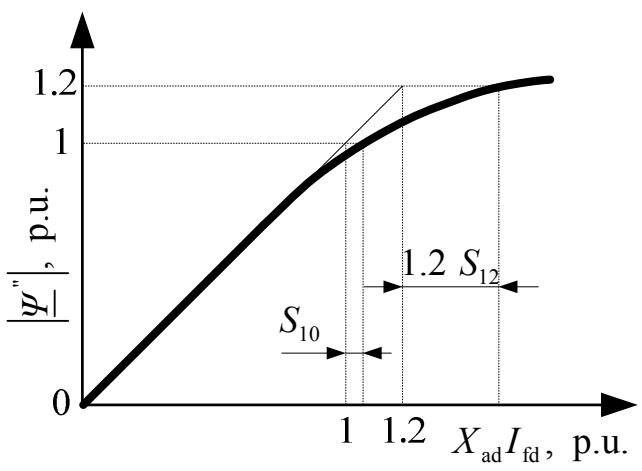

b)

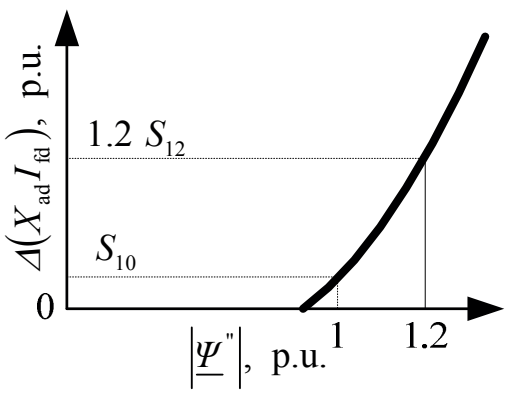

Fig. 1. a) subtransient flux linkage modulus and b) saturation corrections of this flux

defined, by means of which there are expressed the coefficients of a square function approximating relative differences between the nonlinear and linear characteristics of the subtransient flux linkage (Fig. 1b):

$$
\Delta\left(X_{a d} I_{f d}\right)=\alpha(|\underline{\Psi}|-\beta)^{2},
$$


where:

$$
\alpha=\frac{1.2 \cdot S_{12}}{(1.2-\beta)^{2}}, \quad \beta=\frac{\sigma \cdot 1.2-1}{\sigma-1}, \quad \sigma=\sqrt{\frac{S_{10}}{1.2 \cdot S_{12}}}
$$

The electromagnetic torque is given by the formula:

$$
T_{\mathrm{e}}=\Psi_{\mathrm{d}}^{\prime \prime} I_{\mathrm{q}}-\Psi_{\mathrm{q}}^{\prime \prime} I_{\mathrm{d}}
$$

2.2. The model of type RL. In models of a RL-type synchronous generator, the rotor damping circuits of distributed constants are approximated by several equivalent circuits of lumped constants. Denoting the type of the mathematical model of a generator by a pair of numbers $\left(1+n_{\mathrm{d}}, n_{\mathrm{q}}\right)$ - where $n_{\mathrm{d}}, n_{\mathrm{q}}$ determine the number of the equivalent damping circuits in the $\mathrm{d}$ and $\mathrm{q}$ axes of the rotor - there are determined the models of $(3,3),(3,2),(2,2)$, or $(2,1)$ type. The $(2,2)$ model $[2,3,7$, 20-23], when neglecting or taking into account the transformation voltage in the armature circuit $[2,20,21]$, is often used to investigate the PS angular stability. Saturation of the magnetic cores [1] can be taken into account in an approximate way by introducing a correction for the main magnetic flux dependent on the magnetizing current $[2,3,23]$. The effect of the leakage flux saturation, the magnetic hysteresis, and the phenomenon of magnetic cross-coupling between the circuits in the $\mathrm{d}$ and $\mathrm{q}$ axes are then neglected. The models taking into account the saturation in the $\mathrm{d}$ and $\mathrm{q}$ axes base on the magnetization characteristics in each axis. For machines with a uniform air-gap (turbogenerator), it is often assumed that the magnetization characteristic of cores is the same for the $d$ and $q$ axes. For machines with a non-uniform air gap (hydrogenerator), often saturation only in the $\mathrm{d}$ axis is taken into account, because of a large gap in the q axis.

For the turbogenerator model of $(2,2)$ type, when neglecting the transformation voltages in the stator, the machine electrodynamic state is described by the differential equations of the rotor circuits (in relative units):

$$
\begin{gathered}
\frac{\mathrm{d} \Psi_{\mathrm{fd}}}{\mathrm{d} t}=\omega_{\mathrm{N}}\left(E_{\mathrm{fd}}-R_{\mathrm{f}} I_{\mathrm{fd}}\right), \quad \frac{\mathrm{d} \Psi_{\mathrm{Q} 1}}{\mathrm{~d} t}=-\omega_{\mathrm{N}} R_{\mathrm{Q} 1} I_{\mathrm{Q} 1}, \\
\frac{\mathrm{d} \Psi_{\mathrm{D} 1}}{\mathrm{~d} t}=-\omega_{\mathrm{N}} R_{\mathrm{D} 1} I_{\mathrm{D} 1}, \quad \frac{\mathrm{d} \Psi_{\mathrm{Q} 2}}{\mathrm{~d} t}=-\omega_{\mathrm{N}} R_{\mathrm{Q} 2} I_{\mathrm{Q} 2},
\end{gathered}
$$

where: $I_{\mathrm{D} 1}, I_{\mathrm{Q} 1}, I_{\mathrm{Q} 2}, \Psi_{\mathrm{fd}}, \Psi_{\mathrm{D} 1}, \Psi_{\mathrm{Q} 1}, \Psi_{\mathrm{Q} 2}-$ currents and flux linkages in the damping circuits in the $\mathrm{d}$ and q axes referred to the stator side; $R_{\mathrm{f}}, R_{\mathrm{D} 1}, R_{\mathrm{Q} 1}, R_{\mathrm{Q} 2}$ - referred resistances of the rotor electrical circuits; $\omega_{\mathrm{N}}-$ rated angular speed.

Moreover, the following dependencies hold:

$$
\begin{gathered}
\Psi_{\mathrm{fd}}=L_{\sigma \mathrm{f}} I_{\mathrm{fd}}+\Psi_{\mathrm{md}}^{\mathrm{sat}}, \quad \Psi_{\mathrm{Q} 1}=L_{\sigma \mathrm{Q} 1} I_{\mathrm{Q} 1}+\Psi_{\mathrm{mq}}^{\mathrm{sat}}, \\
\Psi_{\mathrm{D} 1}=L_{\sigma \mathrm{D} 1} I_{\mathrm{D} 1}+\Psi_{\mathrm{md}}^{\mathrm{sat}},
\end{gathered}
$$

where: $L_{\sigma \mathrm{f}}, L_{\sigma \mathrm{D} 1}, L_{\sigma \mathrm{Q} 1}, L_{\sigma \mathrm{Q} 2}$ - leakage inductances of the rotor electrical circuits; $\Psi_{\mathrm{md}}^{\mathrm{sat}}, \Psi_{\mathrm{mq}}^{\mathrm{sat}}$ - values of the axial components of the main flux linkage when taking into account the saturation phenomenon.

The saturated main flux linkages in the d and q axes $\left(\Psi_{\mathrm{md}}^{\mathrm{sat}}, \Psi_{\mathrm{mq}}^{\mathrm{sat}}\right)$ are expressed by the flux corrections $\Delta \Psi_{\mathrm{md}}, \Delta \Psi_{\mathrm{mq}}$, (Fig. 2a) $[2,24]$ :

$$
\Psi_{\mathrm{md}}^{\mathrm{sat}}=\Psi_{\mathrm{md}}-\Delta \Psi_{\mathrm{md}}, \quad \Psi_{\mathrm{mq}}^{\mathrm{sat}}=\Psi_{\mathrm{mq}}-\Delta \Psi_{\mathrm{mq}}
$$

where:

$$
\begin{gathered}
\Psi_{\mathrm{md}}=L_{\mathrm{ad}} I_{\mathrm{md}}, \quad \Psi_{\mathrm{mq}}=L_{\mathrm{aq}} I_{\mathrm{mq}}, \\
I_{\mathrm{md}}=-I_{\mathrm{d}}+I_{\mathrm{fd}}+I_{\mathrm{D} 1}, \quad I_{\mathrm{mq}}=-I_{\mathrm{q}}+I_{\mathrm{Q} 1}+I_{\mathrm{Q} 2} .
\end{gathered}
$$

Assuming a proportional reduction of the main magnetic flux in the $\mathrm{d}$ and q axes (Fig. 2b), one obtains:

$$
\begin{gathered}
\Delta \Psi_{\mathrm{md}}=\frac{\Psi_{\mathrm{md}}^{\mathrm{sat}}}{\Psi_{\mathrm{m}}^{\mathrm{sat}}} \Delta \Psi_{\mathrm{m}}=\frac{\Psi_{\mathrm{md}}}{\Psi_{\mathrm{m}}} \Delta \Psi_{\mathrm{m}}, \\
\Delta \Psi_{\mathrm{mq}}=\frac{\Psi_{\mathrm{mq}}^{\mathrm{sat}}}{\Psi_{\mathrm{m}}^{\mathrm{sat}}} \Delta \Psi_{\mathrm{m}}=\frac{\Psi_{\mathrm{md}}}{\Psi_{\mathrm{m}}} \Delta \Psi_{\mathrm{m}},
\end{gathered}
$$

where:

$$
\Delta \Psi_{\mathrm{m}}^{\mathrm{sat}}=\sqrt{\left(\Psi_{\mathrm{md}}^{\mathrm{sat}}\right)^{2}+\left(\Psi_{\mathrm{mq}}^{\mathrm{sat}}\right)^{2}}, \Psi_{\mathrm{m}}=\sqrt{\Psi_{\mathrm{md}}^{2}+\Psi_{\mathrm{mq}}^{2}} .
$$

a)

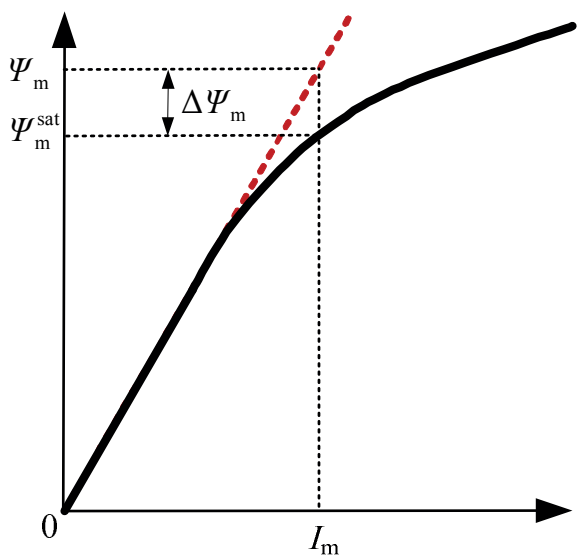

b)

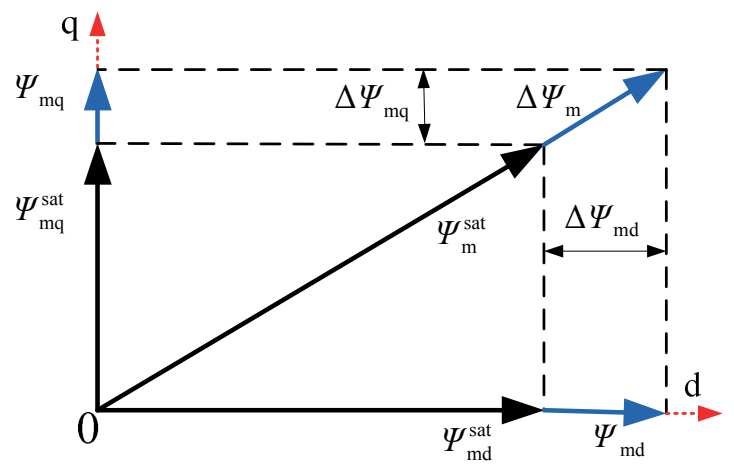

Fig. 2. a) Magnetization characteristic and b) saturation corrections in $\mathrm{d}$ and $\mathrm{q}$ axis of the generator 
The correction $\Delta \Psi_{\mathrm{m}}$ is determined on the basis of the generator no-load characteristic whose nonlinear part is approximated with a square function for the magnetizing current $I_{\mathrm{m}}=\sqrt{I_{\mathrm{md}}^{2}+I_{\mathrm{mq}}^{2}}$. It can be expressed by two coefficients $S_{10}$ and $S_{12}$ (as for the GENROU model) [3, 18, 20].

When neglecting the transformation voltages, the generator stator equations are algebraic equations of the form:

$$
\begin{aligned}
& V_{\mathrm{d}}=-R_{\mathrm{a}} I_{\mathrm{d}}+\omega L_{\sigma} I_{\mathrm{q}}-\omega \Psi_{\mathrm{mq}}^{\mathrm{sat}}, \\
& V_{\mathrm{q}}=-R_{\mathrm{a}} I_{\mathrm{q}}-\omega L_{\sigma} I_{\mathrm{d}}+\omega \Psi_{\mathrm{md}}^{\mathrm{sat}}
\end{aligned}
$$

The generator electromagnetic torque $T_{\mathrm{e}}$ is given by the formula:

$$
T_{\mathrm{e}}=\Psi_{\mathrm{d}} I_{\mathrm{q}}-\Psi_{\mathrm{q}} I_{\mathrm{d}}
$$

Equation (4) for the electromagnetic torque of the model GENROU is equivalent to (11) if there exists the subtransient symmetry $X_{\mathrm{d}}^{\prime \prime}=X_{\mathrm{q}}^{\prime \prime}$.

2.3. Equations of mechanical motion. The equations of mechanical motion (in relative units) have the form of:

$$
\frac{\mathrm{d} \omega}{\mathrm{d} t}=\frac{1}{T_{\mathrm{J}}}\left(T_{\mathrm{m}}-T_{\mathrm{e}}\right), \quad \frac{\mathrm{d} \delta}{\mathrm{d} t}=\omega_{\mathrm{N}}(\omega-1),
$$

where: $\omega$ - rotor angular speed, $\delta$ - load angle, $T_{\mathrm{J}}-$ mechanical time constant, $T_{\mathrm{m}}$ - turbine mechanical torque.

\section{Power rejection tests}

The measurement results of selected quantities in steady and transient states, caused by opening the main switch connecting a generating unit with the power system, resulting in generator power rejection (Fig. 3), can be the basis of estimation of synchronous generator model parameters. The main advantage of such a disturbance is the elimination of the influence of an

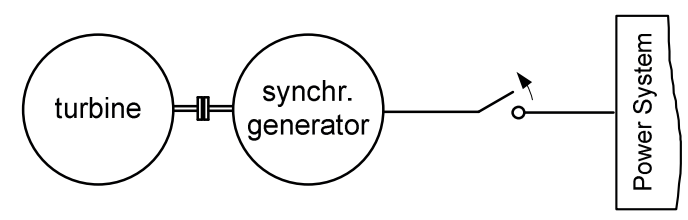

Fig. 3. Load rejection test of the synchronous generator driven by a turbine

outer power system on the generating unit under investigation $[3,7,17,18,24,25]$.

By selecting the appropriate generator operating conditions before performing a power rejection test, one can cause for the transient phenomena to occur only in one generator axis. Thanks to that, parameter estimation can be simplified and made for the $\mathrm{d}$ and $\mathrm{q}$ generator axes separately.

For power rejection in the $\mathrm{d}$ axis, the generator has to be initially (that is before opening the switch) loaded with only small reactive power $\left(P_{0}=0, Q_{0} \neq 0\right)$. Such initial load causes that for the duration of the transient state (initiated by opening the switch) there occur only the stator current component in the $\mathrm{d}$ axis, the stator voltage (induced in the $\mathrm{q}$ axis), and the excitation current change, while the rotor speed is constant. Fig. 4a shows the position of the phasors of the terminal generator voltage $\left(\underline{V}_{\mathrm{T} 0}\right)$ and current $\left(\underline{I}_{\mathrm{T} 0}\right)$ on the complex plane before the disturbance.

For power rejection in the $\mathrm{q}$ axis, the generator has to be loaded with only small active power, while such generator reactive power is chosen that the current phasor $\left(\underline{I}_{\mathrm{T} 0}\right)$ lies along the $\mathrm{q}$ axis, and the load angle $\delta_{0}=-\varphi_{0}$, where $\varphi_{0}$ is the power coefficient angle (Fig. 4b) $[3,18,25,26]$. In this case, the generator's initial active power $P_{0} \neq 0$ and reactive power $Q_{0} \neq 0$. Power rejection in the $\mathrm{q}$ axis results in changes of the stator voltage in the $d$ axis and the excitation current, as well as, due to the saturation, in small changes of the voltage in the $q$ axis. That is why the machine circuits, in both $\mathrm{d}$ and $\mathrm{q}$ axes, are taken into account in the calculations.

In the mathematical model of the generator, the generator power rejections are represented by step changes of the stator current axial components from the initial values to zero. a)

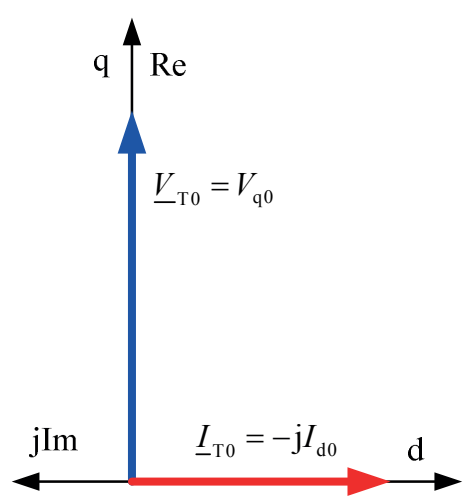

b)

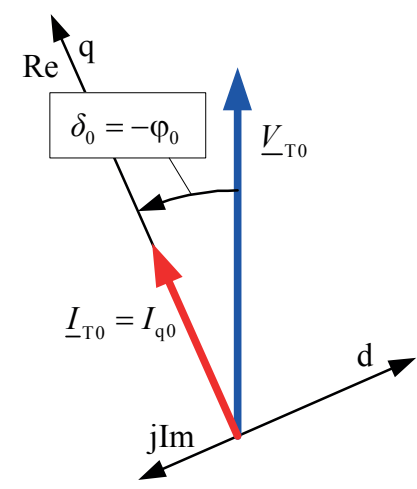

c)

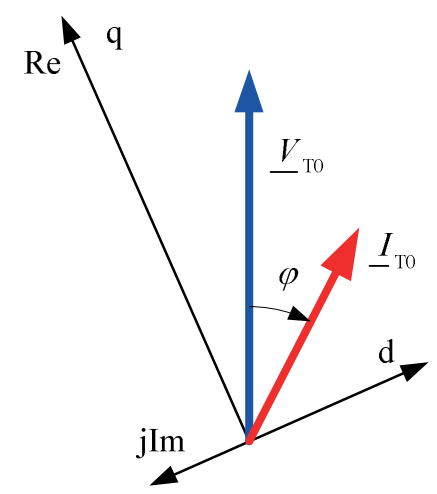

Fig. 4. Phasors of terminal voltage and current for power rejection a) in d axis, b) in q axis, and b) at arbitrary power rejection 
Opening the main switch when loading the generator with active and reactive power for which the conditions of power rejection in the $\mathrm{d}$ and $\mathrm{q}$ axes are not met, corresponds to arbitrary active and reactive power rejection (Fig. 4c) [3, 24].

When taking measurements in a power plant, difficulties can appear concerning the accurate determination of the initial values of the generator's active and reactive power corresponding to the power rejection in the q axis. These values can often be determined with only a certain accuracy. Also, the staff of a power plant may not agree to introduce the appropriate value of the generator's initial active power. Opening the main switch under such conditions corresponds to arbitrary active and reactive power rejection. In this case, the electrical circuits of the generator's longitudinal and transverse axes influence the disturbance waveforms. The generator parameters in the $\mathrm{d}$ and $\mathrm{q}$ axes can be estimated through the analysis of the disturbance waveforms at the arbitrary power rejection. It can also be assumed that the generator parameters in the $d$ axis were determined before (e.g. through the analysis of the waveforms at power rejection in the $\mathrm{d}$ axis) and the generator parameters in the $\mathrm{q}$ axis are the searched ones.

\section{Initial conditions of state equations for parameter estimation of the synchronous generator model}

In the generator model parameter estimation process, the generator differential state equations are repeatedly solved for current values of parameters. Initial values of the state variables can be determined for the state before the disturbance for a steady operating point of the generator, determined by active power $P_{0}$, reactive power $Q_{0}$, and stator voltage $V_{0}$.

4.1. The model of type XT. The initial values of the state variables for the GENROU model were determined under a simplifying assumption of the machine's subtransient symmetry $X_{\mathrm{d}}^{\prime \prime}=X_{\mathrm{q}}^{\prime \prime}=X^{\prime \prime}$. In this case, the equivalent diagram of the generator stator in the steady state is of the form shown in Fig. 5. The complex plane was selected so that the voltage phasor $\underline{V}_{0}$ was real.

The complex power, voltage, and current of the generator are given by:

$$
\begin{gathered}
\underline{S}_{0}=P_{0}+\mathrm{j} Q_{0}, \\
\underline{V}_{0}=V_{0}, \quad \underline{I}_{0}=\frac{P_{0}-\mathrm{j} Q_{0}}{V_{0}} .
\end{gathered}
$$

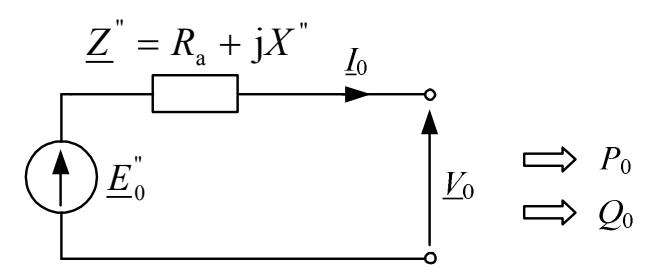

Fig. 5. The steady-state equivalent circuit of the generator at subtransient symmetry
The voltage phasor behind the subtransient impedance of the generator is expressed by:

$$
\underline{E}_{0}^{\prime \prime}=\left(R_{\mathrm{a}}+\mathrm{j} X X^{\prime \prime}\right) \underline{I}_{0}+\underline{V}_{0},
$$

and the voltage phasor behind the subtransient impedance together with a saturation correction is given by:

$$
\underline{E}_{0 \text { sat }}^{\prime \prime}=\left(\left|\underline{E}_{0}^{\prime \prime}\right|+\left|\Delta \underline{E}_{0}^{\prime \prime}\right|\right) \mathrm{e}^{\mathrm{j} \operatorname{Arg}\left\{\underline{E}_{0}^{\prime \prime}\right.} .
$$

The generator power angle is:

$$
\delta_{0}=\operatorname{Arg}\left\{\underline{E}_{\mathrm{Q}}\right\},
$$

where:

$$
\underline{E}_{\mathrm{Q} 0}=\underline{E}_{0 \text { sat }}^{\prime \prime}+\left(X_{\mathrm{q}}-X^{\prime \prime}\right) \underline{I}_{0},
$$

If the real axis coincides with the $\mathrm{q}$ axis (as in Fig. 4), the current and generator terminal voltage phasors are given by:

$$
\underline{I}_{\mathrm{T} 0}=\underline{I}_{0} e^{-\mathrm{j} \delta_{0}}, \underline{V}_{\mathrm{T} 0}=\underline{V}_{0} e^{-\mathrm{j} \delta_{0}},
$$

and the initial values of the stator current and flux linkages in the $\mathrm{d}$ and $\mathrm{q}$ axes are:

$$
\begin{gathered}
I_{\mathrm{d} 0}=\operatorname{Im}\left\{\underline{I}_{\mathrm{T} 0}^{*}\right\}, I_{\mathrm{q} 0}=\operatorname{Re}\left\{\underline{I}_{\mathrm{T} 0}^{*}\right\}, \\
\Psi_{\mathrm{d} 0}^{\prime \prime}=\operatorname{Re}\left\{\underline{E}_{0}^{\prime \prime} e^{-\mathrm{j} \delta_{0}}\right\}, \Psi_{\mathrm{q} 0}^{\prime \prime}=-\operatorname{Im}\left\{\underline{E}_{0}^{\prime \prime} e^{-\mathrm{j} \delta_{0}}\right\} .
\end{gathered}
$$

The initial values of the state variables are determined by the relationships:

$$
\begin{aligned}
& E_{\mathrm{q} 0}^{\prime}=\Psi_{\mathrm{d} 0}^{\prime \prime}+I_{\mathrm{d} 0}\left(X_{\mathrm{d}}^{\prime}-X^{\prime \prime}\right), \\
& E_{\mathrm{d} 0}^{\prime}=\Psi_{\mathrm{q} 0}^{\prime \prime}+I_{\mathrm{q} 0}\left(X_{\mathrm{q}}^{\prime}-X^{\prime \prime}\right), \\
& \Psi_{\mathrm{D} 10}=E_{\mathrm{q} 0}^{\prime}-I_{\mathrm{d} 0}\left(X_{\mathrm{d}}^{\prime}-X_{\sigma}\right), \\
& \Psi_{\mathrm{Q} 10}=E_{\mathrm{d} 0}^{\prime}-I_{\mathrm{q} 0}\left(X_{\mathrm{q}}^{\prime}-X_{\sigma}\right),
\end{aligned}
$$

The initial conditions for load rejection in the $\mathrm{d}$ axis are obtained based on $(13) \div(16)$ when assuming $P_{0}=0$ and $I_{\mathrm{q} 0}=0$. In the case of load rejection in the q axis, the generator should be loaded with active power $P_{0}$ and reactive power $Q_{0}$, for which $\delta_{0}=-\varphi_{0}$. At a given generator voltage $V_{0}$ and active power $P_{0}$, reactive power $Q_{0}$ can be determined by solving the nonlinear equation:

$$
\operatorname{tg} \varphi_{0}=\frac{Q_{0}}{P_{0}}=-\operatorname{tg} \delta_{0}
$$

by iterative method when taking into consideration $(13) \div(16)$ [3]. 
4.2. The model of type RL. From the nonlinear system of algebraic equations

$$
\begin{gathered}
P_{0}=V_{\mathrm{d} 0} I_{\mathrm{d} 0}+V_{\mathrm{q} 0} I_{\mathrm{q} 0}, \\
\frac{\sqrt{P_{0}^{2}+Q_{0}^{2}}}{V_{0}}=\sqrt{I_{\mathrm{d} 0}^{2}+I_{\mathrm{q} 0}^{2}}, \quad V_{\mathrm{d} 0}=R I_{\mathrm{d} 0}+\omega L_{\mathrm{q}} I_{\mathrm{q} 0},
\end{gathered}
$$

one can determine the axial components of the stator current and voltage $I_{\mathrm{d} 0}, I_{\mathrm{q} 0}, V_{\mathrm{d} 0}, V_{\mathrm{q} 0}$.

The field current referred to the stator side is given by:

$$
I_{\mathrm{fd} 0}=\frac{1}{\omega L_{\mathrm{ad}}}\left(V_{\mathrm{q} 0}-R I_{\mathrm{q} 0}-\omega L_{\mathrm{d}} I_{\mathrm{d} 0}\right) .
$$

Given that the currents in the generator damping circuits are equal to zero in the steady state:

$$
I_{\mathrm{D} 1}=I_{\mathrm{Q} 1}=I_{\mathrm{Q} 2}=0,
$$

one can determine from $(6) \div(9)$ the initial values of the flux linkages as the state variables of (5).

Initially, approximate values of the resistance and inductance of the windings, given e.g. in the catalog data of a generator, can be used in the relationships determining the initial values of the state variables for the generator models of XT and RL type. The current values of the parameters are used in subsequent iterations of parameter estimation.

\section{Estimation of synchronous generator model parameters}

The method of parameter estimation of the generator model consists, in approximation, of the waveforms measured with the functions expressed by the searched parameters of the model. The measured signals are strongly disturbed, so they must be appropriately filtered. The used zero-phase filtration with a third-order digital Butterworth filter [27] gave satisfactory results.

The least squares method can be used for parameter estimation of particular synchronous generator models. In the approximation process, these parameters are selected in such a way that the objective function determined in the form of the mean square error, defined for deviations (determined at the $i$-th instants) between the waveforms measured and those calculated based on the mathematical models, is minimized [3, $16,18,26,28-30]$

$F(\boldsymbol{X})=\sum_{i=1}^{n}\left(\left|V_{\mathrm{T} i(\mathrm{~m})}-V_{\mathrm{T} i(\mathrm{~s})}(\boldsymbol{X})\right|^{2}+\left|I_{\mathrm{fd} i(\mathrm{~m})}-I_{\mathrm{fd} i(\mathrm{~s})}(\boldsymbol{X})\right|^{2}\right)$,

where: index $\mathrm{m}$ denotes the measured waveform of excitation current and terminal generator voltage and index $\mathrm{s}$ denotes the simulated waveform of these quantities, calculated for the vector of the searched parameters $\boldsymbol{X}$.

Minimization of the objective function (21) is a nonlinear problem which can be solved by numerical methods. The Newton gradient algorithm was used to minimize the objective function (21). This algorithm is a deterministic local minimization algorithm. It requires that the objective function $F(\boldsymbol{X})$ should be a real function having continuous derivatives of first and second order in a given search interval. The gradient and Hessian of this function are used to search for the minimum of the function $F(X)$ [13-15]. Fig. 6 shows a simplified flowchart of the Newton gradient algorithm.

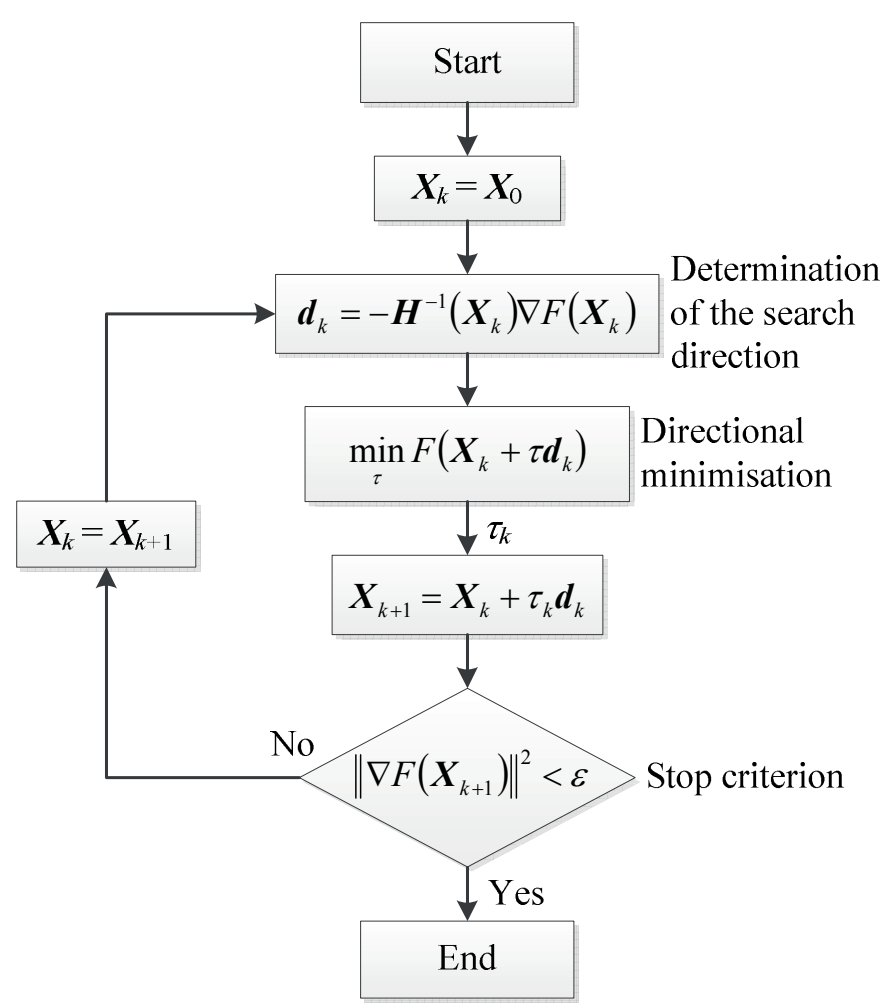

Fig. 6. Flowchart of the Newton gradient algorithm [12]

In the zero-iteration of the algorithm (for $k=0$ ), the read is the starting point $\boldsymbol{X}_{0}$ and parameters of the algorithm, i.a. the calculation accuracy $\varepsilon$. Next, the values of the gradient and Hessian of the function $F$ in the successive iteration point $\boldsymbol{X}_{k}$ are calculated. On this basis, there is determined the search direction vector $\boldsymbol{d}_{k}$. Then, the directional minimization in the direction of the vector $\boldsymbol{d}_{k}$ aimed at selection of the step-size coefficient $\tau_{k}$ is performed. After each iteration, the stop condition, where $\|\bullet\|$ denotes the vector norm, is checked. If the stop condition is met or if the permissible number of iterations is exceeded, the algorithm is ended. In the algorithm, there are also taken into account the limitations of the search intervals of particular objective functions [12-15]. 


\section{Calculation results}

The calculations of the parameters of the analyzed mathematical models of the generator were based, first of all, on the measured dynamic waveforms of the excitation voltage (generator input signal), as well as on the stator voltage and excitation current (output signals) at the power rejection in the $\mathrm{d}$ axis for $Q_{0}=0.5531$ in the generating unit No. 5 in Rybnik Power Plant $[3,30]$. In the analyzed generating unit, there is installed a TWW-200-2A turbogenerator (of ratings: $S_{\mathrm{N}}=235 \mathrm{MV} \cdot \mathrm{A}$, $\left.V_{\mathrm{TN}}=15.75 \mathrm{kV}, \cos \varphi_{\mathrm{N}}=0.85\right)$ driven by a steam turbine. This generator is equipped with an electromachine excitation system. Fig. 7 shows the measured waveform of the excitation voltage, determined by the impact of the generator voltage regulation system. Figures 8 and 9 show the waveforms of the terminal voltage and the excitation current, measured and calculated for the resultant values of the parameters for the GENROU and RL $(3,3)$ and $(2,2)$ models. The resultant values of the parameters of these models are given in Table 1. For comparison, the parameters of the GENROU model, recalculated from the parameters of the RL $(2,2)$ model, are also presented in Table 1.

Table 1

Parameters of the models RL $(3,3),(2,2)$ and GENROU calculated based on the waveforms recorded during power rejection in the $\mathrm{d}$ axis in Rybnik Power Station and parameters XT determined based on the parameters RL of the model $(2,2)$

\begin{tabular}{|c|c|c|c|c|c|}
\hline Par. & RL33 & RL22 & Par. & RL33 & RL22 \\
\hline$R$ & 0.00190 & 0.00190 & $R_{\mathrm{D} 2}^{\bullet}$ & 0.00765 & - \\
\hline$L_{\sigma}$ & 0.1192 & 0.1181 & $L_{\sigma \mathrm{f}}^{\bullet}$ & 0.07774 & 0.1293 \\
\hline$L_{\mathrm{ad}}$ & 2.0965 & 2.1707 & $R_{\mathrm{f}}^{\bullet}$ & 0.00102 & 0.00134 \\
\hline$L_{\mathrm{\sigma D} 1}^{\bullet}$ & 0.00023 & 0.00021 & $S_{10}$ & 0.00189 & 0 \\
\hline$R_{\mathrm{D} 1}^{\bullet}$ & 0.00823 & 0.00626 & $S_{12}$ & 0.1119 & 0.1124 \\
\hline$L_{\mathrm{\sigma D} 2}^{\bullet}$ & 0.09006 & - & & & \\
\hline
\end{tabular}

\begin{tabular}{|c|c|c|c|c|c|}
\hline Par. & GENROU & $\begin{array}{c}\text { GENROU } \\
\text { from RL22 }\end{array}$ & Par. & $\begin{array}{c}\text { GENROU } \\
\text { from RL22 }\end{array}$ & RL22 \\
\hline$R$ & 0.00190 & 0.00190 & $T_{\mathrm{do}}^{\prime}$ & 5.5767 & 5.4494 \\
\hline$X_{\sigma}$ & 0.1274 & 0.1181 & $T_{\mathrm{do}}^{\prime \prime}$ & 0.0600 & 0.0621 \\
\hline$X_{\mathrm{d}}$ & 2.2865 & 2.2888 & $S_{10}$ & 0.00105 & 0 \\
\hline$X_{\mathrm{d}}^{\prime}$ & 0.2426 & 0.2402 & $S_{12}$ & 0.1029 & 0.1124 \\
\hline$X_{\mathrm{d}}^{\prime \prime}$ & 0.1278 & 0.1183 & & & \\
\hline
\end{tabular}

Next, the waveforms obtained at the arbitrary power rejection in Halemba Heat and Power Plant for $P_{0}=0.08042$ and $Q_{0}=0.05509$ were analyzed [31]. In the analyzed generating unit, an 6H6372/2 turbogenerator manufactured by Škoda was installed (of ratings: $P_{\mathrm{N}}=50 \mathrm{MW}, V_{\mathrm{TN}}=10,5 \mathrm{kV}$ ), driven by a steam turbine. This generator is equipped with a SEMIPOL

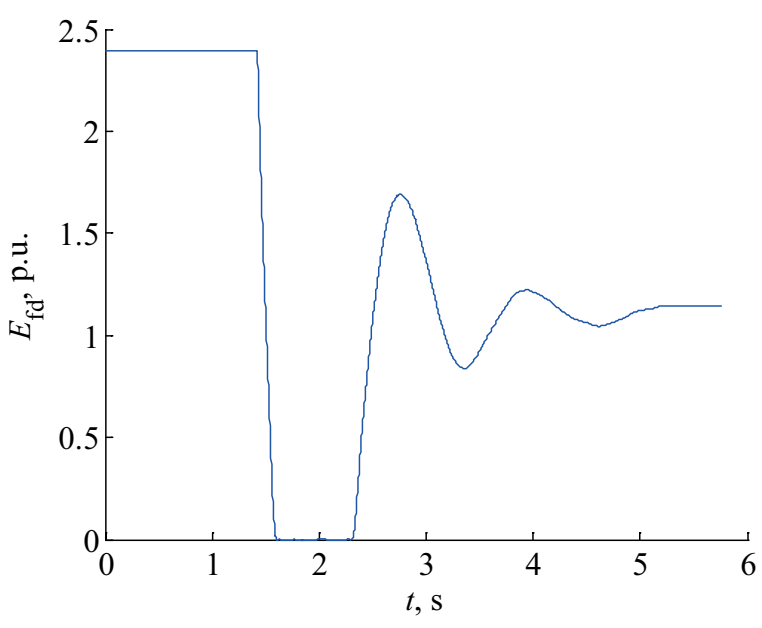

Fig. 7. The measured waveforms of the of the excitation voltage for power rejection in the $\mathrm{d}$ axis in Rybnik Power Station

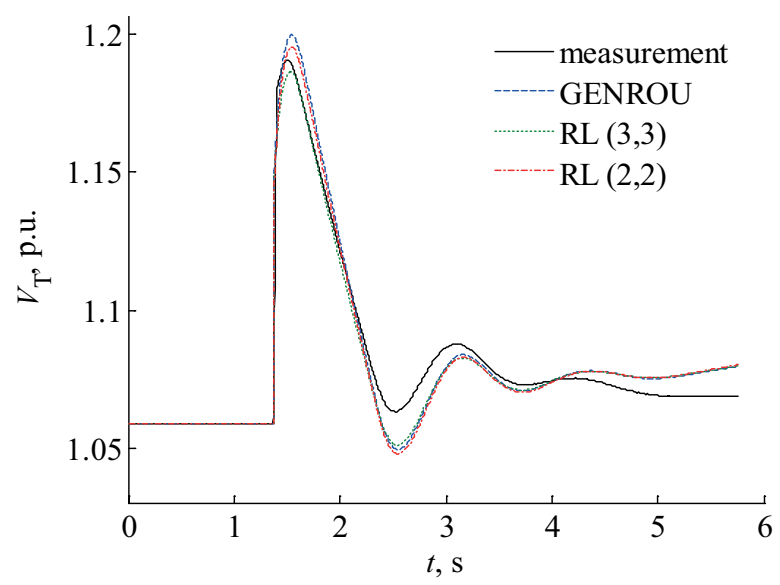

Fig. 8. Waveforms of the excitation current for power rejection in the $\mathrm{d}$ axis in Rybnik Power Station, measured and calculated for the resultant values of parameters for the models: GENROU, RL $(3,3)$ and $(2,2)$

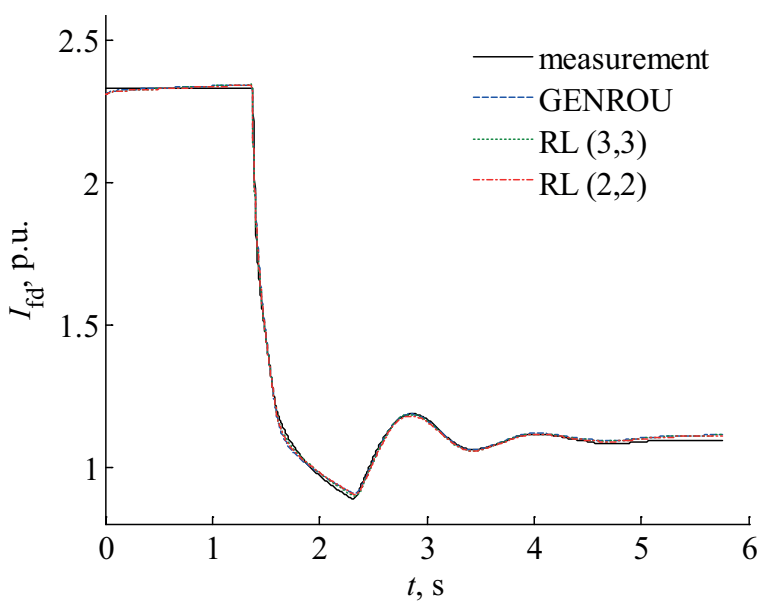

Fig. 9. Waveforms of the excitation current for power rejection in the $\mathrm{d}$ axis in Rybnik Power Station, measured and calculated for the resultant values of parameters for the models: GENROU, RL $(3,3)$ and $(2,2)$ 


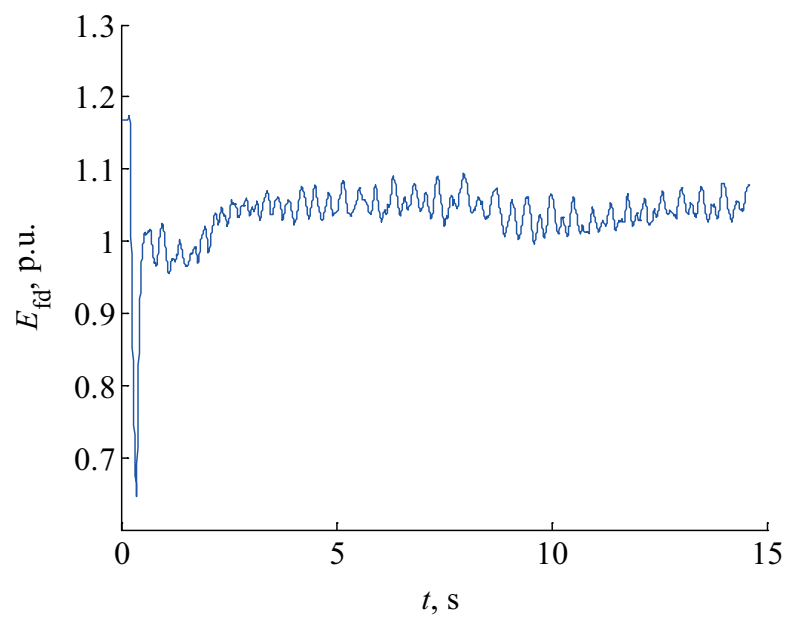

Fig. 10. The measured waveforms of the excitation voltage for power rejection in the d axis in Halemba Heat and Power Plant

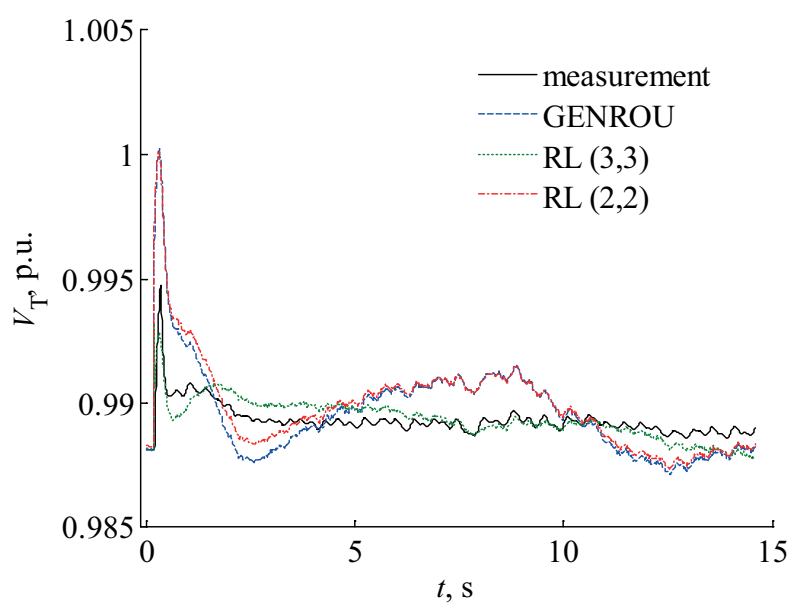

Fig. 11. Waveforms of the voltage at generator terminals for arbitrary power rejection in Halemba Heat and Power Plant, measured and calculated for the resultant values of parameters for the models: GENROU, RL $(3,3)$ and $(2,2)$

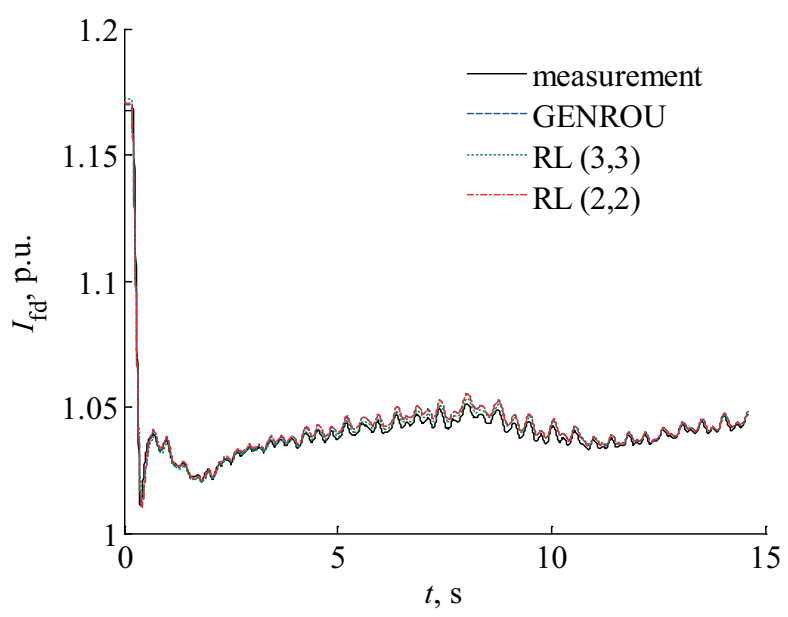

Fig. 12. Waveforms of the excitation current for arbitrary power rejection in Halemba Heat and Power Plant, measured and calculated for the resultant values of parameters for the models: GENROU, RL $(3,3)$ and $(2,2)$ static excitation system. Fig. 10 shows the waveforms of the excitation voltage, Figs. 11 and 12 present the waveforms of the terminal voltage and the excitation current measured and calculated for the resultant values of the parameters for the models: GENROU, RL $(3,3)$ and $(2,2)$. The resultant values of the parameters of these models are given in Table 2 .

\section{Table 2}

Parameters of the models RL $(3,3),(2,2)$ and GENROU calculated based on the waveforms recorded during arbitrary power rejection in Halemba Heat and Power Plant and parameters XT, determined based on the parameters RL of the model $(2,2)$

\begin{tabular}{|c|c|c|c|c|c|}
\hline Par. & RL33 & RL22 & Par. & RL33 & RL22 \\
\hline$R$ & 0.00088 & 0.00088 & $L_{\mathrm{aq}}$ & 1.6756 & 1.5722 \\
\hline$L_{\sigma}$ & 0.1538 & 0.1549 & $L_{\sigma \mathrm{Q} 1}^{\bullet}$ & 2.5190 & 0.7516 \\
\hline$L_{\mathrm{ad}}$ & 1.6526 & 1.6868 & $R_{\mathrm{Q} 1}^{\bullet}$ & 0.00561 & 0.00463 \\
\hline$L_{\sigma \mathrm{D} 1}^{\bullet}$ & 0.03306 & 0.00307 & $L_{\sigma \mathrm{Q} 2}^{\bullet}$ & 0.01194 & 0.00134 \\
\hline$R_{\mathrm{D} 1}^{\bullet}$ & 0.00701 & 0.00429 & $R_{\mathrm{Q} 2}^{\bullet}$ & 0.04884 & 0.01265 \\
\hline$L_{\sigma \mathrm{D} 2}^{\bullet}$ & 0.07326 & - & $L_{\sigma \mathrm{Q} 3}^{\bullet}$ & 0.09280 & - \\
\hline$R_{\mathrm{D} 2}^{\bullet}$ & 0.00649 & - & $R_{\mathrm{Q} 3}^{\bullet}$ & 0.01135 & - \\
\hline$L_{\sigma \mathrm{\sigma}}^{\bullet}$ & 0.07339 & 0.1555 & $S_{10}$ & 0.07310 & 0.07260 \\
\hline$R_{\mathrm{f}}^{\bullet}$ & 0.00095 & 0.00134 & $S_{12}$ & 0.2346 & 0.2341 \\
\hline
\end{tabular}

\begin{tabular}{|c|c|c|c|c|c|}
\hline Par. & GENROU & $\begin{array}{c}\text { GENROU } \\
\text { from RL22 }\end{array}$ & Par. & GENROU & $\begin{array}{c}\text { GENROU } \\
\text { from RL22 }\end{array}$ \\
\hline$R$ & 0.00088 & 0.00088 & $X_{\mathrm{q}}$ & 1.6502 & 1.7271 \\
\hline$X_{\sigma}$ & 0.1476 & 0.1549 & $X_{\mathrm{q}}^{\prime}$ & 0.7152 & 0.6634 \\
\hline$X_{\mathrm{d}}$ & 1.8417 & 1.8417 & $X_{\mathrm{q}}^{\prime \prime}$ & 0.1450 & 0.1562 \\
\hline$X_{\mathrm{d}}^{\prime}$ & 0.2966 & 0.2973 & $T_{\mathrm{qo}}^{\prime}$ & 1.647 & 1.5960 \\
\hline$X_{\mathrm{d}}^{\prime \prime}$ & 0.1450 & 0.1579 & $T_{\mathrm{q}}^{\prime \prime}$ & 0.1615 & 0.1282 \\
\hline$T_{\mathrm{do}}^{\prime}$ & 4.3979 & 4.3517 & $S_{10}$ & 0.07235 & 0.07260 \\
\hline$T_{\mathrm{do}}^{\prime \prime}$ & 0.1040 & 0.1079 & $S_{12}$ & 0.2265 & 0.2341 \\
\hline
\end{tabular}

\section{Conclusions}

In the paper, parameter estimation results of the synchronous generator nonlinear models on the basis of power rejection tests when using the Newton gradient algorithm were presented. In the calculations, the load rejection test realized for a generator operating in a power plant was used. The realization of the test is relatively simple contrary to e.g. tests for a machine working under no-load conditions which require disconnecting the generator from the power system and its long driving $[3,11,16]$. Furthermore, the test of power rejection is a large disturbance in the steady state, despite its simplicity and safety for the 
generator operation. This test causes great changes in voltages and currents which are influenced by all the parameters of the model, i.e. the parameters in the $\mathrm{d}$ and $\mathrm{q}$ axes. As a result, based on the recorded waveforms it is easier to determine parameters of the model, as opposed to tests using a small disturbance (e.g. step or pseudorandom small changes of the reference voltage in the generator voltage regulation channel).

Based on the investigations performed, it can be stated that:

- It is possible to estimate the parameters of the synchronous generator XT and RL models from measurements of the appropriate dynamic waveforms at power rejection.

- The parameter estimation was made through minimization of a complicated, nonlinear objective function (21). This function was determined in the form of a mean square error between selected measurement (on a real object) waveforms and calculated (based on the assumed model, initial conditions, and current parameters) waveforms. The Newton local optimization gradient algorithm with constraints was used to minimize the objective function.

- The nonlinearity of magnetic core magnetization should be taken into account during the estimation.

- Taking into account the estimation results of the generator RL $(2,2)$ model parameters, one can determine (based on algebraic formulas) the parameters of the model GENROU of type XT (and vice versa).

- The calculation results of the synchronous generator mathematical model parameters should be the basis for performing reliable simulation investigations of a power system.

Summing up, one can state that the errors of the parameter estimation of different synchronous generator models performed with the use of appropriate measurements are not large. Meanwhile, the use of the generator parameters determined based on the catalog data for simulations of steady and transient states in a power system results in considerable calculation errors. Large errors are also caused by using a model that does not take into account the saturation of the magnetic circuit. Fig. 13 shows exemplary waveforms obtained for the model GENROU

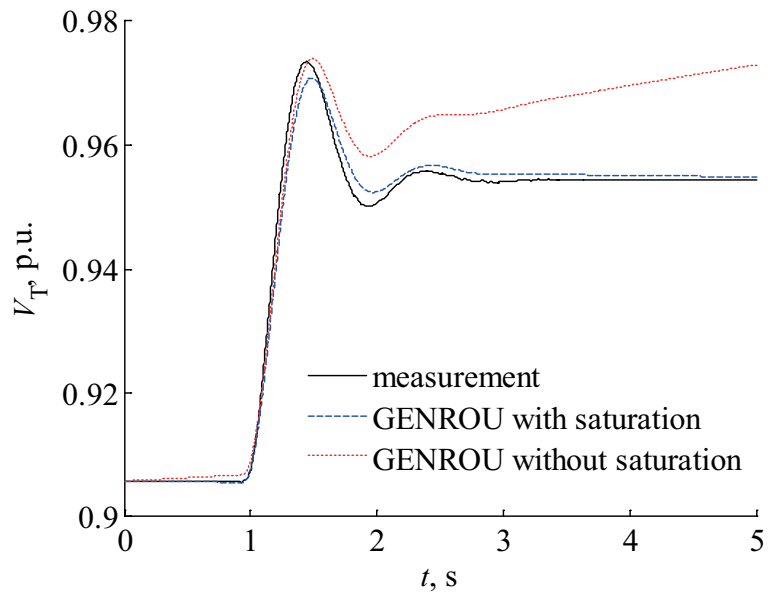

Fig. 13. Exemplary waveforms obtained for the GENROU model taking and not taking into account the saturation of the magnetic circuit taking and not taking into account the saturation of the magnetic circuit (with the same parameters XT) at a step change in the reference voltage of the generator operating under no-load, compared with the measured waveforms. The influence of not taking into account the saturation of the magnetic circuit of generators increases when analysing and simulating an expanded, multi-machine PS (there occur larger differences between the actual waveforms and the simulation results).

\section{REFERENCES}

[1] L. Gołębiowski, M. Gołębiowski, and D. Mazur, "Magnetic non-linearity in 3D FEM models of the electric machines", Academic Journals of Poznan University of Technology 5, 95-111 (2007).

[2] 1110-2002 IEEE Guide for Synchronous Generator Modeling Practices and Application in Power System Stability Analyses, IEEE, 2003.

[3] S. Paszek, S. Berhausen, A. Boboń, Ł. Majka, A. Nocoń, M. Pasko, P. Pruski, and T. Kraszewski, Measurement estimation of dynamic parameters of synchronous generators and excitation systems working in the National Power System, Monograph 504, (2013), [in Polish].

[4] A.M. El-Serafi and A.S. Abdallah, "Effect of saturation on the steady-state stability of a synchronous machine connected to an infinite bus system", IEEE Trans. Energy Conversion 6 (3), 514-521 (1991).

[5] M.A. Arjona., C. Hernandez, M. Cisneros-Gonzalez, and R. Escarela-Perez, "Estimation of synchronous generator parameters using the standstill step-voltage test and a hybrid genetic algorithm", Electrical Power and Energy Systems 35, 105-111 (2012).

[6] H. Bissig, K. Reichert, and T.S. Kulig, "Modelling and identification of synchronous machines, a new approach with an extended frequency range", IEEE Trans. Energy Conversion 8 (2), 263-271 (1993).

[7] M. Ghomi and Y. Najafi Sarem, "Review of synchronous generator parameters estimation and model identification", 42nd International Universities Power Engineering Conference UPEC, 228-235 (2007).

[8] M. Hasni, O. Touhami, R. Ibtiouen, M. Fadel, and S. Caux, "Estimation of synchronous machine parameters by standstill tests", Mathematics and Computers in Simulation 81, 277-289 (2010).

[9] M. Huang, W. Li, and W. Yana, "Estimating parameters of synchronous generators using square-root unscented Kalman filter", Electric Power Systems Research 80, 1137-1144 (2010).

[10] S. Berhausen and S. Paszek, "Use of the finite element method for parameter estimation of the circuit model of a high power synchronous generator", Bull. Pol. Ac.: Tech. 63 (3), 575-582 (2015).

[11] Ł. Majka and S. Paszek, "Mathematical model parameter estimation of a generating unit operating in the Polish National Power System", Bull. Pol. Ac.: Tech. 64 (2), 409-416 (2016).

[12] P. Pruski and S. Paszek, The modal analysis of selected disturbance waveforms in a power system. Determination of angular stability factors, Monograph 592, (2016), [in Polish].

[13] L. Rade and B. Westergren, Mathematics Handbook for Science and Engineering, Springer-Verlag, Berlin, 2004.

[14] J. Bonnans, J. Gilbert, C. Lemarechal, and C. Sagastizábal, Numerical Optimization. Theoretical and Practical Aspects, Springer-Verlag, Berlin, 2006. 
[15] A. Kaw, E. Kalu, and D. Nguyen, Numerical Methods with Applications, University of South Florida, Tampa, 2011.

[16] A. Boboń, S. Paszek, P. Pruski, T. Kraszewski, and M. Bojarska, "Computer-aided determining of parameters of generating unit models based on measurement tests", Przeglad Elektrotechniczny 5, 17-21 (2011).

[17] F.P. de Mello and L.H. Hannett, "Validation of Synchronous Machine Models and Derivation of Model Parameters from Tests", IEEE Transaction on Power Apparatus and Systems 100 (2), 662-672 (1981).

[18] S. Paszek, A. Boboń, J. Kudła, J. Bialek, and N. Abi-Samra, "Parameter estimation of the mathematical model of a generator, excitation system and turbine", Przeglad Elektrotechniczny 11, 7-12 (2005)

[19] S. Paszek and A. Nocoń, Optimisation and Polyoptimisation of Power System Stabilizer Parameters, LAMBERT Academic Publishing, Saarbrücken, 2014.

[20] P.C. Krause, Analysis of Electric Machinery, McGraw-Hill Book Company, New York, 1986.

[21] P. Kundur, Power System Stability and Control, McGraw-Hill, Inc., New York, 1994

[22] P.M. Anderson and A.A. Fouad, Power System Control and Stability, John Wiley \& Sons, New York, 2003.

[23] O. Chee-Mun, Dynamic Simulation of Electric Machinery using Matlab/Simulink, Prentice Hall - PTR, New Jersey, 1998.

[24] E.C. Bortoni and J.A. Jardini, "Identification of Synchronous Machine Parameters using Load Rejection Test Data", IEEE Transaction on Energy Conversion 17 (2), 242-247 (2002).
[25] J.W. Feltes, S. Orero, B. Fardanesh, E. Uzunovic, S. Zelingher, and N. Abi-Samra, "Deriving Model Parameters from Field Test Measurements", IEEE Computer Applications in Power 15 (4), 30-36 (2002).

[26] A. Boboń, M. Bojarska, T. Kraszewski, Ł. Majka, M. Pasko, S. Paszek, and P. Pruski, "Computations of generating unit model parameters using program PARZW with database", 10th International Conference CONTROL OF POWER SYSTEMS, 169-170 (2012).

[27] A.V. Oppenheim and R.W. Schafer, Digital Signal Processing, Prentice Hall, Englewood Cliffs, 1974.

[28] M. Dehghani, M. Karrari, W. Rosehart, and O.P. Malik, "Synchronous machine model parameters estimation by a time-domain identification method", Electrical Power and Energy Systems 32, 524-529 (2010).

[29] E. Mouni, S. Tnani, and G. Champenois, "Synchronous generator modelling and parameters estimation using least squares method", Simulation Modelling Practice and Theory 16, 678-689 (2008).

[30] S. Paszek and $Ł$. Majka, "Computations of the model parameters of generating unit elements based on measurements", $A T \& P$ Journal PLUS2 2008, 49-53 (2008).

[31] A. Nocoń, A. Boboń, S. Paszek, M. Pasko, P. Pruski, Ł. Majka, D. Szuster, and M. Bojarska, "Measurement parameter estimation of the model of a synchronous generator working in thermal electric power plant", 10th International Conference on Advanced Methods in the Theory of Electrical Engineering, AMTEE, VI-3-4 (2011). 